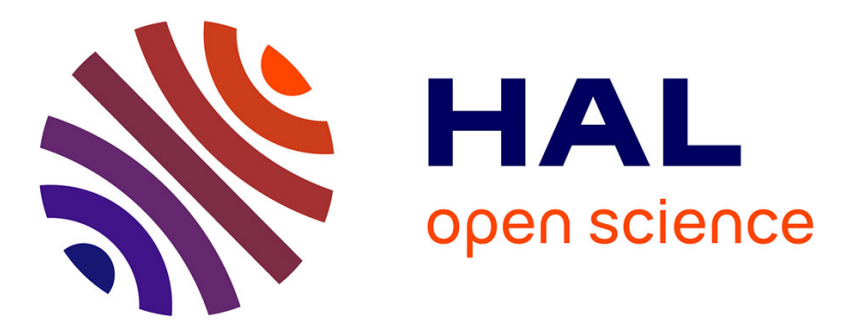

\title{
Le XXe siècle chez les montagnards et les Vietnamiens, raconté par Jacques Dournes à Andrew Hardy (Bagard, France - 1er et 2 février 1992)
}

Andrew Hardy, Jacques Dournes

\section{- To cite this version:}

Andrew Hardy, Jacques Dournes. Le XXe siècle chez les montagnards et les Vietnamiens, raconté par Jacques Dournes à Andrew Hardy (Bagard, France - 1er et 2 février 1992). Aséanie, Sciences humaines en Asie du Sud-Est, 2009, 24, pp.149-171. halshs-02566512

\section{HAL Id: halshs-02566512}

https://shs.hal.science/halshs-02566512

Submitted on 23 Feb 2022

HAL is a multi-disciplinary open access archive for the deposit and dissemination of scientific research documents, whether they are published or not. The documents may come from teaching and research institutions in France or abroad, or from public or private research centers.
L'archive ouverte pluridisciplinaire HAL, est destinée au dépôt et à la diffusion de documents scientifiques de niveau recherche, publiés ou non, émanant des établissements d'enseignement et de recherche français ou étrangers, des laboratoires publics ou privés. 
Le XXe siècle chez les montagnards et les Vietnamiens Raconté par Jacques Dournes à Andrew Hardy (Bagard, France, 1er et 2 février 1992)

Jacques Dournes, Andrew Hardy

\section{Citer ce document / Cite this document :}

Dournes Jacques, Hardy Andrew. Le XXe siècle chez les montagnards et les Vietnamiens Raconté par Jacques Dournes à Andrew Hardy (Bagard, France,1er et 2 février 1992). In: Aséanie 24, 2009. pp. 149-171;

doi : https://doi.org/10.3406/asean.2009.2110

https://www.persee.fr/doc/asean_0859-9009_2009_num_24_1_2110

Fichier pdf généré le 08/07/2021 


\title{
Le $\mathrm{XX}^{\mathrm{e}}$ siècle chez les montagnards et les Vietnamiens
}

\author{
Raconté par Jacques Dournes à Andrew Hardy
}

(Bagard, France $-1^{\text {er }}$ et 2 février 1992)

JACQUES DOURNES - Maintenant, on ne fait plus de thèses d'État, on fait des thèses d'université! Ce n'est pas difficile de faire une thèse. Vous soutenez n'importe quelle connerie devant un jury qui n'y connaît rien du tout: vous êtes le seul à connaître la question et vous partez avec la mention très bien. Pas compliqué. Il suffit d'avoir un directeur de thèse qui est de connivence.

Mais moi, je peux me vanter d'une thèse d'État en Sorbonne, avec Lévi-Strauss au jury. Il y avait foule dans l'amphithéâtre de la Sorbonne. C'était bourré. Je me suis bien marré. De tous les membres du jury, Lévi-Strauss était le seul qui avait lu ma thèse et c'est le seul avec lequel j'ai discuté. Il m'attaquait sur des problèmes d'analyse structurale. Il m'attaquait, je lui répondais, c'était du ping-pong. C'était brillant de tous les côtés, magnifique. C'était le 23 juin 1973. Ça fait presque vingt ans. J'étais déjà vieux, hein! Cinquante ans. À l'époque, c'était rare de soutenir une thèse d'État avant cinquante ans. J'ai soutenu la mienne un an après Condominas, un an après Haudricourt et tant d'autres. Donc j'étais un des plus jeunes à soutenir une thèse d'État. C'était sérieux à ce moment-là. d'État?

ANDREW HARDY - Et la thèse de Gourou, Les paysans du delta, c'était une thèse

J.D. - Pour moi, c'est l'ouvrage qui fait référence actuellement. Gourou était un grand bonhomme, un homme droit, parfaitement droit. Je ne suis pas géographe. Je ne connais pas le Tonkin - ce qu'on appelait le Tonkin en ce temps-là, ce qu'on a appelé ensuite le Nord Viêt-nam. Mais il me semble difficile quand même de parler des paysans du delta en s'en tenant à l'[agri]culture. Il y a [aussi] l'homme, ce qu'il fait, dans sa maison, sa langue. Et Gourou, il ne parlait pas vietnamien [et il n'a jamais su ce que c'était] que de tremper les pieds nus dans la rizière. 
Moi, j'en suis là. Si on n'a pas été pieds nus dans la rizière, on ne sait rien, parce que tout se passe dans la tête des gens. L'économique, l'agronomique, ce sont des épiphénomènes et ce n'est pas ça qui explique les phénomènes. Que ce soit Gourou pour le Tonkin, que ce soit Delvert pour le Cambodge, que ce soient beaucoup d'autres, ils n'ont pas trempé dans la boue. Ce sont des sociologues. Il y a des chiffres, il y a des rendements à l'hectare, il y a des trucs comme ça. Pour moi, ça n'a aucun intérêt (mais je ne dis pas que pour les économistes ça n'en ait pas). L'intérêt pour moi, c'est l'homme vis-à-vis de son milieu, ce qu'il va en faire, ce qu'il va en sortir, en diachronie, en suivant le fil des siècles et l'évolution des politiques et de la psychologie des hommes. Comment l'homme, pieds nus dans la rizière avec de la boue jusqu'à mi-jambes, réfléchit, réagit, pense et rêve à autre chose aussi - c'est ça qui est intéressant. Le rendement à l'hectare, je m'en fous, je n'ai jamais mesuré ça. Je suis anthropologue, je ne suis pas géographe.

$\infty 000000000$

J.D. - Vous me parliez de dates [à propos de votre sujet de thèse]. Vous me parliez de 1950 à 1960. Ça ne correspond à rien. Vous savez très bien que les dates historiques ne sont pas les dates de siècle. Le XXe siècle a commencé en 1919 et le XXI a commencé en 1991. Ce sont les événements humains qui fabriquent les époques, les dates et les séquences. Alors pour l'Indochine, si vous voulez prendre une tranche, [il vaudrait mieux choisir] 1946-1954 - un petit travail historique que personne n'ose franchement aborder.

A. H. - La guerre d'Indochine...

J.D. - Mais non! C'est du temps des Américains qu'il y eut la guerre. Je parle du temps des Français. Je connais à fond. J'ai débarqué en Indochine en 1946 et j'ai été foutu à la porte en 1954 ! Je connais à fond, de tous les côtés: côté français, côté vietnamien, côté des minorités. Et je vous garantis que de 1946 à 1954, on ne faisait pas la guerre. La guerre, elle a commencé en 1954.

A. H. - On faisait quoi?

J.D. - C'était... c'était de la comédie. De la comédie! Les Français voulaient faire du fric et on trichait de tous les côtés.

Il faut connaître aussi toute l'histoire qui s'est passée juste avant 1946. De 1940 à 1945, l'Indochine française était sous la coupe de l'amiral Decoux, pétainiste à bloc, comme tous les chefs de province, les évêques, tous les Français. L'Indochine était donc pétainiste et pro allemande. Par conséquent, pas hostile aux Japonais.

En 1945, coup de force japonais en Indochine. Decoux et compagnie sont complètement cocufiés: les Japs prennent le pouvoir, envoient les Français en camp de concentration et ont cette idée géniale de donner leur indépendance aux 
Vietnamiens. L'indépendance du Viêt-nam vient de la grâce du Mikado! C'est historiquement vrai!

En 1946 de Gaulle décide la reconquête militaire de l'Indochine. Alors, au printemps-été 1946, il y a quelques bateaux chargés de militaires qui quittent Marseille pour aller à Saïgon, pour la reconquête. Moi, j'ai fait partie du premier bateau civil, en septembre-octobre 1946 donc je suis absolument contemporain des événements.

À ce moment-là (décembre 1946), Hô Chi Minh, qui n'était pas une canaille, a dit: «Que les Français nous envoient des instituteurs mais pas des militaires!». Je l'ai entendu. Moi, petit jeune - j'avais vingt-quatre ans - je déclare en pleine assemblée de tous ces curés, évêques, administrateurs et tout ça: «Hô Chi Minh a raison, la culture française mais pas la bataille ». Oh, je me suis fait bien voir dès le début! Hô Chi Minh n'en a jamais rien su, mais moi j'en ai reçu plein les gencives. Il avait raison: il se trouve qu'aujourd'hui, il n'y a pas un Vietnamien qui parle français au Viêt-nam.

Par-dessus le marché, ce de Gaulle nomme comme gouverneur général Thierry d'Argenlieu - un religieux carme déchaussé ${ }^{1}$ qu'on appelait aussi « Ruolz », parce que [le ruolz était un métal argenté dans lequel on fabriquait des couverts qui] « [tenaient] lieu d'argenterie ». Il était la risée de tout le monde, Thierry d'Argenlieu.

A. H. - D'Argenlieu a renvoyé en France beaucoup de pétainistes d'Indochine?

J.D. - Quasi tous. Jusqu'à l'évêque de Saïgon, le dernier Français évêque de Saïgon, qui était chef de la légion pétainiste en Indochine, c'est-à-dire chef de tous les Français pétainistes!

J.D. - Je vais vous donner une généralité sur la vie économique. C'est que pendant toute la période française - la période des méchants colonialistes, c'est-àdire celle que j'ai connue de 1946 à 1954 - tout le monde mangeait bien. Les routes étaient circulables; le train de Saïgon à Hanoï, ça roulait ; et les gens bouffaient bien.

1. Rappelons qu'en France, l'ordre du Carmel est scindé en deux grandes branches: les carmes et carmélites déchaussés (ou déchaux) issus de la réforme de sainte Thérèse d'Ávila et de saint Jean de la Croix d'une part, et les grands carmes et carmélites chaussés, qui n'ont pas adopté cette réforme, d'autre part. Les carmes déchaussés tiendraient leur nom du fait qu'ils portaient des sandales très ouvertes ou qu'ils étaient pieds nus dans leurs sandales.

Officier de marine, Thierry d'Argenlieu (1889-1964) avait quitté la flotte pour entrer chez les carmes en 1920. Mobilisé en 1939, il avait rejoint les forces de la France Libre dès 1940. Nommé Haut Commissaire de France en Indochine par le général de Gaulle en 1945, il s'y montra partisan d'un retour inconditionnel à la domination française, refusant tout dialogue avec le gouvernement provisoire dirigé par Hô Chi Minh. Il reprit la vie monastique en 1948. 
Oh... il y avait les escarmouches en brousse (le Viêt-minh) contre les Français, contre je ne sais pas quoi. Mais c'était des escarmouches, rien de plus.

Le Viêt-nam, c'est un pays franchement riche. Je n'ai jamais vu quelqu'un avoir faim, même sous la guerre américaine. Sauf dans les camps de concentration - parce que les Américains avaient fait des camps de concentration pas seulement pour les Vietnamiens, les soi-disant communistes, mais pour les minorités ethniques, pour les montagnards (les Moïs) - et là-dedans on crevait de faim copieusement. Mais dans les villages - j'avais une quarantaine de villages dans un rayon de dix kilomètres autour de chez moi, une vallée très riche - je n'ai jamais vu quelqu'un avoir faim, jamais!

Le Viêt-nam exportait du riz - oui - alors que depuis l'époque américaine le Viêt-nam importe du riz de Thaillande. Ça en dit long. Et pourquoi? Parce que l'empire stalinien de Hanoï réquisitionnait tout le riz pour l'envoyer en Russie, sous prétexte que les Russes avaient aidé les Vietnamiens à prendre leur indépendance - dette de guerre! Mais parce que, surtout, sous l'époque américaine, les chars, les quelques chars vietnamiens, les quelques avions vietnamiens - tout l'armement - étaient entièrement russes. Ce qui est marrant c'est que cet armement transitait par la Chine, qui détestait les Russes mais qui fermait les yeux. Les Vietnamiens ont envoyé tout leur riz en Russie jusqu'à l'époque de Gorbatchev. Résultat: plus de riz pour les paysans vietnamiens.

À partir du départ des Français, mais surtout après 1975 (c'est à partir de 1975 que ça a été la catastrophe pour le Viêt-nam), c'est là qu'il y a eu les boat people. Le régime de Hanoï a fait la réunification du Viêt-nam. À la différence de la Corée (par exemple), ce n'était pas une évidence historique. La Corée, c'est un problème uniquement politique et américain - il n'y a jamais eu deux Corées dans l'histoire, c'est comme l'Allemagne. Tandis que le Viêt-nam a toujours eu un royaume du Nord et un royaume du Sud. Et ils ne se sont jamais aimés. Ils ont des dialectes tels que quelqu'un de Hanoï et quelqu'un de Saïgon ont du mal à se comprendre (sans parler des gens de Hué qui parlent encore un autre dialecte) - façon de mettre les accents, les tons. Ils se comprennent tout juste. Au téléphone ils ne se comprennent pas du tout. Il faut écrire pour se comprendre!

À partir de 1975 donc, le régime de Hanoï a imposé ses structures, notamment économiques. Il n'y avait plus de propriété privée. Tous les travailleurs donnaient tout à l'État. L'État leur donnait trois conneries pour trois cents tonnes. C'est la même chose que ce qui s'est passé en Russie, qui est un pays extrêmement riche. Ça regorge de tout, la Russie. C'est une stupidité d'envoyer de l'aide alimentaire actuellement tant qu'elle n'est pas distribuée, pour la bonne raison qu'il n'y a pas de réseau de distribution. Il faut demander à tel bureau, qui va demander à tel bureau, qui va demander à tel bureau, puis entre deux bureaux il y a un flemmard qui oublie de téléphoner et ça reste des mois comme ça - patates en train de pourrir, etc., etc. C'est ça, le régime. 
Dégoûtés de ne plus avoir de propriété privée, dégoûtés de devoir tout donner à l'État, les paysans vietnamiens - et aussi montagnards - ont produit de moins en moins. Résultat: ils n'ont rien à bouffer. Les riches importent du riz de Thaillande, alors que c'était l'inverse quand j'étais au Viêt-nam.

\section{$\infty 000000000$}

A. H. - Est-ce que la guerre d'Indochine a eu un grand impact sur l'économie?

J. D. - Il faut bien distinguer entre la guerre française et la guerre américaine. La guerre française, c'était pour défendre une colonie - une colonie de peuplement -, ce que les Américains n'ont jamais fait. Des Français, des familles entières - des planteurs - s'installaient et restaient des décennies. Ils s'intéressaient au pays. Ils aimaient la terre, ils faisaient souche, quelquefois avec une femme vietnamienne, et ça ne marchait pas si mal.

Ce n'était pas si terrible, la colonisation. Oh, il y avait des bagnes, Poulo Condore - quelques dizaines de gars qu'on appelait communistes et qu'on a fourrés au bagne - mais il n'y a pas eu de scènes horribles. Sur le plan économique, les Français avaient des plantations - caoutchouc, thé, café - qui ne profitaient qu'aux Européens. Parce que les Vietnamiens ne se servaient pas de ce caoutchouc, les Vietnamiens ne boivent pas de café et ils ont tellement de thé. Les plantations étaient uniquement pour le fric des planteurs français.

A. H. - Et les gens qui travaillaient, les Vietnamiens?

J.D. - La plupart étaient des gens du Nord. Parce que dans le Sud, il y a beaucoup de rizières, les rizières très riches de la Cochinchine. Alors ils travaillaient dans la rizière. Il y avait surplus de riz, parce qu'on en vendait partout. J'ai toujours connu le Viêt-nam comme exportateur de riz. Mais il fallait des coolies sur les plantations et comme les montagnards - c'est leur caractère, ils sont anarchistes ! - ne voulaient pas travailler pour un patron, on a fait venir des Vietnamiens du Nord. Et ils travaillaient bien.

A.H. - Ils en ont profité?

J. D. - Ils n'étaient pas malheureux. Ils étaient payés, ils étaient nourris, ils avaient des logements, ils n'étaient pas malheureux. Il y avait une espèce de boutique dans la plantation où ils pouvaient acheter - pris sur leur salaire - des vêtements, du tabac, des bricoles. Il n'y avait pas d'argent comme au village.

A.H. - Ils ne pouvaient pas acheter au marché?

J. D. - Les plantations étaient éloignées des centres villes. C'était en brousse : il n'y avait aucun transport en commun. Une fois que le pauvre coolie vietnamien du Nord s'installait là-bas il ne bougeait plus. C'était eux le marché !

A. H. - Mais en 1945, après le coup de force, ils ont tous quitté les plantations. 
J.D. - Il n'y en avait pas beaucoup en 1945, des gens du Nord. Non, c'est après 1946 que ça a commencé. Avant c'était des petites entreprises familiales, pas de gros gros machins, sinon Michelin. Mais Michelin, c'était le caoutchouc et c'était limitrophe - entre Viêt-nam et Cambodge. En fait, les plus grosses plantations étaient sur le Cambodge, province de Mondolkiri. Les plantations se sont développées entre 1946 et 1954. Un développement énorme! Je l'ai vu. De jour en jour, j'ai vu les progrès. J'ai vu des planteurs français, fusil à la main, aller dans mes villages, obliger mes... « Moïs » à venir travailler sur leurs plantations. J'ai porté plainte. L'administrateur français m'a soutenu. Il a été vidé, envoyé en Afrique. Et moi quelques années après, j’ai été vidé. Parce que les planteurs, la Banque d'Indochine et Bao Dai, tout ça c'était la même chose! J'étais appelé « le curé rouge » par Bao Dai. Il a exigé mon rapatriement.

Donc, les Vietnamiens du Nord sont venus sur les plantations. Puis ils ont construit leur maison, ils y ont fait souche et ils y sont toujours. C'est eux qui ont les plantations maintenant, avec un apport chinois, il ne faut pas oublier. Le patron, bien sûr, est chinois. Ce sont les Chinois qui ont remplacé les Français comme patrons des grandes plantations.

A. H. - C'est toujours les Chinois, maintenant? Après 1978-1979?

J. D. - Je manque d'informations. Mais tant que j'y étais, c'étaient des Chinois qui étaient les gros patrons et des Vietnamiens du Nord qui travaillaient, pas malheureux du tout. Tout ça faisait la richesse du Viêt-nam: thé, café, caoutchouc. Surtout thé et café - le caoutchouc a baissé régulièrement parce qu'on a fait du caoutchouc synthétique tout simplement. Thé et café, ça marchait vraiment bien, sur le plan économique.

Quant aux montagnards, quand on leur foutait la paix, ils mangeaient bien! Pensez: sur les hauts plateaux - mon cher pays - la densité était de huit au kilomètre carré (alors qu'à Hanoï, la densité était de quatre-vingts à quatre-vingt-dix au kilomètre carré)! C'était du luxe! On grattait un peu la terre, on mettait quelques grains de riz et on en récoltait plein, plein, plein - et du bon riz encore! On bouffait vraiment bien, il n'y a aucun problème.

A.H. - Et dans le delta du Sud?

J. D. - Dans le delta du Mékong, une terre très très riche, on faisait trois récoltes de riz par an. C'était bourré d'alluvions : ça déboulait, ça déboulait... ça va boucher la mer. Finalement, l'Indochine va rejoindre la Malaisie si ça continue comme ça. Dans deux mille ans, ça sera peut-être le cas.

0000000000

J.D. - Il y a deux économies. Il y a l'économie interne. C'est-à-dire comment les gens vivent - les paysans vietnamiens comme les paysans montagnards: des petites exploitations familiales, une terre ultra-riche. Je parle d'avant 1954, tant que 
c'était la soi-disant guerre française. Je pouvais aller chez n'importe quel paysan: j'étais nourri comme ça, je me régalais! Des petits poissons, des crabes de rizière, un tas de trucs délicieux, du vrai nước mắm! Le paysan vietnamien et le montagnard se ressemblent énormément. Ils n'ont jamais été ennemis (on a inventé des trucs là-dessus). C'était à qui roulerait le plus l'autre, ça oui! Mais ça marchait très bien. Il n'y avait pas de rixe, il n'y avait pas de bataille, il n'y avait rien.

Voilà pour l'économie interne. Quant à l'économie externe, les exportations étaient considérables - thé, café, caoutchouc - et procuraient des devises énormes au Viêt-nam. Donc tout était très équilibré.

A. H. - Et les Vietnamiens avaient un rôle important dans cette économie?

J. D. - Les Chinois surtout.

A. H. - Les Vietnamiens, ils étaient...

J. D. - Des exécuteurs. Jusqu'en 1954, tout le monde vivait bien. Tout le monde avait dans la maison de quoi manger tous les jours et de quoi nourrir dix gosses. Je dis bien, dix gosses - c'est une moyenne dans la famille. Là j'ai fait des statistiques chez les Jörai. Moyenne de naissances: dix par mariage. Survivants : sept, au bout de trois ans. Pas mal, hein? Ce qui fait que les Vietnamiens, qui étaient à peine quarante millions en 1946, sont maintenant quatre-vingt-dix millions, beaucoup plus nombreux que les Français sur un territoire beaucoup plus petit. Normal qu'ils envahissent le Laos et le Cambodge. Quand un Américain descendait un Vietnamien pendant la guerre, une femme vietnamienne mettait au monde deux gosses. C'était coulé d'avance, la politique militaire américaine. Ça pond, ça pond, ça pond et puis tout le monde travaille. À quatre ans, le gosse commence à travailler dans le jardin. On cultive les ruisseaux, le bord du chemin, on cultive tout. On met des patates douces, on met du piment, on met des arachides, on met tout, jusqu'au bord de la route. C'est des travailleurs infatigables, increvables les Vietnamiens. Alors là, pour le travail je les respecte. Qu'est-ce qu'ils travaillent!

Il n'y a qu'à voir à Paris : les boat people, ils arrivent dans le XIII e arrondissement, sans un sou. Ils commencent par faire la plonge dans un restaurant vietnamien. Ça dure six mois maximum. Après ça ils servent à table. Après ça ils aident à la cuisine. Et en moins d'un an, ils ouvrent un restaurant à côté. Ils font fortune. Chapeau pour le travail. Et par-dessus le marché, c'est bon ce qu'ils font. C'est sain, c'est frais, c'est bon, c'est propre. Personne n'a été malade d'un plat vietnamien. Les Vietnamiens se sont imposés. J'ai entendu un commerçant vietnamien à Paris, place Maubert, me dire: «Ah! Paris, c'est notre plus belle colonie! »Ce n'est pas beau ça? Eux qui colonisent la France à leur tour, avec beaucoup d'humour et beaucoup d'amitié pour la France. Joli retour des choses, sans lutte, sans rien de violent, mais en s'imposant par le travail et la qualité.

Alors vous voyez, l'économie familiale interne a très bien fonctionné, tant que c'était l'anarchie. Parce que les Français ne vérifiaient pas ce qui se passait dans les villages. Hanoï était très loin et n'avait pas encore beaucoup de visées sur le Sud. Donc 
c'était pratiquement ce qu'on appelait le « communisme » vietnamien, c'est-à-dire [la prépondérance] de la commune. En ce temps-là c'était impeccable. Tout le monde en a de très bons souvenirs. Parce que les Français n'ont pas vraiment voulu se battre.

Quand j'étais jeune, j'étais comme mon petit chien, j'étais fureteur, j'allais partout, j'étais dans les camps militaires, dans les campements, dans les petits bastions perdus dans la forêt. Je voyais des Français paumés, à trois ou quatre, avec quelques montagnards autour. Une frousse épouvantable: ils n'osaient pas sortir de leur poste. Ils étaient ravitaillés par hélicoptère, ha! Ils ne voulaient pas se battre. Ils ne se battaient pas! Il a fallu quelques généraux absurdes pour vouloir organiser une grande bataille dans le nord du Viêt-nam, pour reprendre un Viêt-nam qui leur avait déjà échappé.

A. H. - Ils voulaient quand même défendre leurs intérêts économiques.

J.D. - Pas les militaires, ils s'en foutaient. Les militaires et les planteurs ne pouvaient pas se voir. Bah! Il n'y avait pas de collusion française là-dedans, pas du tout.

A. H. - Et les industriels, les planteurs, comment ils s'en sortaient?

J. D. - Peinards comme tout! Personne n'allait sur leurs plantations. De temps en temps, le Viêt-minh qui allait descendre un pauvre coolie pour faire peur. Pas grand-chose, jusqu'en 1954. Des bricoles. Non, ce n'était pas vraiment la guerre. Les planteurs français étaient très contents.

A. H. - Ils pensaient rester?

J.D. - Oui, tout le monde pensait rester. Moi aussi. Bon... Ils avaient leurs ennemis, moi, j'avais les miens, mais tout le monde pensait rester.

A. H. - Et les Chinois dans tout ça?

J. D. - Oh les Chinois, dans l'intérieur pas grand-chose. Jusqu'en 1954, ce n'était pas encore développé. Mais c'est eux qui avaient la mainmise sur l'import-export à Saïgon.

A.H. - Comment le riz allait-il des rizières jusqu'à Chợ Lớn?

J. D. - Ça dépend des époques. Jusqu'en 1954, il y avait la consommation domestique et puis il y avait le ramasseur chinois qui prenait le surplus et qui allait le vendre sur le port de Saïgon. Il habitait Chợ Lớn et il avait des petits Chinois qui travaillaient pour lui, qui allaient ramasser.

A. H. - Donc il avait tout un réseau.

J.D. - Oui, c'était des réseaux tout ça. Ce qu'on appelait les congrégations chinoises. Ce qui est très marrant aussi, c'est que tous ces Chinois - qui n'étaient pas pour Tchang Kaï-chek et encore moins pour Mao Tsé-toung - avaient dans leur maison le portrait de Sun Yat-sen, le père commun des deux branches. C'est ravissant!

[Thé, café, riz, tout ce qu'il y avait à vendre était ainsi acheminé sur Saïgon et conservé dans de grands entrepôts aux mains des Chinois.] Quand une denrée commen- 
çait à manquer, ceux-ci bloquaient l'entrepôt et les prix montaient, montaient... Puis ils les débloquaient et vendaient [au prix fort]!

En 1955-1956, j'ai vu un coup fumant avec des commerçants chinois dans le petit village de Choreo chez les Jörai. À mon arrivée, il n'y avait là que le village jörai, ma maison en bordure, la route et quelques commerçants vietnamiens. Quelques Jörai ont essayé de faire un peu de commerce. Des petites bricoles de rien du tout: des boîtes de conserves, des allumettes, etc. Mais ça n'a pas marché du tout, parce que les Jörai sont des philosophes, des artistes, des poètes. Ils achetaient un truc une piastre, ils voulaient le revendre deux pour avoir immédiatement de l'argent. Les Vietnamiens, eux, se débrouillaient à peu près.

Arrivent quelques Chinois... Pendant un mois ou deux, ils vendent à perte ce qui leur coûte deux piastres, ils le revendent une piastre - et en deux mois ils coulent complètement les Vietnamiens et deviennent les maîtres du marché. Après, ils ont fait monter les prix comme ils ont voulu. Trois ans plus tard, il y avait une école chinoise à Choreo devant le village, pour tous les enfants chinois qui étaient là. C'est ça, les Chinois: savoir vendre à perte pour couler l'autre. Ils ont réussi, ils ont coulé tout le monde. Les Vietnamiens, les Jörai, ils ont tout coulé. Comme ils avaient leur congrégation à Saïgon, [ils disposaient du capital nécessaire pour faire de telles manœuvres].

Le commerce international fonctionnait de la même façon. C'est l'époque où il y avait encore des cargos - français surtout, italiens, quelquefois anglais -, peut-être un par an, qui amenaient des produits au port de Saïgon. Ça n'existe plus maintenant. Les Chinois achetaient la cargaison avant de savoir ce qu'il y avait dedans. Ils achetaient tout le bateau. Les Français voulaient avoir ce qu'on appelle le connaissement: savoir ce qu'il y avait à bord et puis acheter telle partie ou telle partie. Le Chinois, lui - le gros Chinois cossu, l'amant de Marguerite Duras - se ramenait là-dedans et disait « moi, j'achète tout, tout! » Il savait très bien que c'était parfaitement vendable. Parce que les cargos de telle travée, ils ne transportaient pas de la cochonnerie. La cargaison allait dans les entrepôts des Chinois à Chợ Lớn. Quand les demandes commençaient à monter pour tel produit, les Chinois n'écoulaient que par petites quantités. Et quand ça devenait la pénurie, alors hop! ils vendaient tout à des prix exorbitants. Ils ont fait des fortunes foireuses - et ça, aucun Vietnamien n'a pu être de taille.

A. H. - Parce que les Vietnamiens avaient la même idée que les Jörai?

J. D. - Oui, petit bénéfice immédiat. Parce que finalement le Vietnamien et le montagnard - je parle des paysans, des gens de l'intérieur, je ne parle pas des bourgeois, pas des citadins - ils sont tellement proches, psychologiquement. Culturellement totalement différents, mais psychologiquement ce sont des petits exploitants de petits lopins de terre. Ils s'entraidaient bien sur place, pas de gros problèmes, pas d'animosité raciale, non. Les Vietnamiens bourgeois, citadins, communistes sont devenus racistes. Les racistes, ils traitent de mọi les sauvages des hauts plateaux, mes amis. Ce que les Grecs appelaient les barbaroi. 
A.H. - Qu'est-ce qu'il y avait comme commerce entre villages?

J. D. - Ça dépend. Entre Vietnamiens pas grand-chose, parce qu'ils récoltent les mêmes produits dans leurs petites exploitations : quelques légumes, plantes marâ̂chères, arbres fruitiers. À l'inverse, chez les montagnards, les Moï, les Jörai, Sré et compagnie, c'est complètement spécialisé. Il y en a qui font des poteries, il y en a qui font des vanneries, il y en a qui font des tissages. Il y en a qui font de la forge, parce que le minerai de fer, ils forgent très très bien. Et on échange. Ça circule tout le temps, tout le temps là-dedans.

A. H. - Et qui fait l'échange?

J.D. - Les privés. Il n'y a pas de Chinois là-dedans. Ce sont des privés entre eux.

A.H. - Donc il n'y avait pas de commerçants?

J.D. - Du tout! du tout!

A.H. - Ils utilisaient une monnaie?

J.D. - Non. Le troc! Ça n'existait pas la monnaie. Je parle des hauts plateaux.

A.H. - C'était sur une petite distance?

J.D. - Oh... ça pouvait aller jusqu'à plus de cent kilomètres! Il y a des Jörai qui faisaient deux cents kilomètres pour filer un buffle à des Lao et se procurer une jarre ou un gong en échange. Deux cents kilomètres!

A. H. - Parce qu'ils savaient qu'il y avait des jarres et des gongs là-bas?

J.D. - Il n'y en a pas sur la partie Viêt-nam [de l'Indochine]. C'est de l'autre côté. Ils faisaient de grandes distances. Tous les montagnards des hauts plateaux qui allaient sur la côte - cent cinquante kilomètres, par ce qu'on appelait les pistes du sel, toutes ces pistes qui allaient grosso modo de l'est vers l'ouest, des montagnes vers la côte - [troquaient] des cornes de rhinocéros, des cochons, des tissages contre du nước mắm, du sel ou du poisson salé qu'ils ramenaient chez eux. Il y a des siècles que ça durait ce machin-là. C'est archiconnu, c'est raconté par beaucoup de littérateurs étrangers bien sûr. Ça circulait beaucoup et à l'amiable, gentiment, à qui volera le plus l'autre (on est en Asie et on n'a pas de carnets, de comptes, de chèques). Ça marchait très bien.

A.H. - Ils se déplaçaient comment?

J.D. - À pied! Si on traîne des cochons derrière soi, il ne faut pas une semaine pour aller des hauts plateaux sur la côte. Si on va en marche forcée, on peut le faire en trois jours. Cent cinquante kilomètres, ce n'est pas terrible, je l'ai fait.

A.H. - Qu'est-ce qu'ils ont comme chaussures?

J. D. - Ils n'en ont pas. Les Vietnamiens non plus, je parle de ceux de la campagne. Tout le monde est pieds nus. On suit des pistes. Quand on sort des pistes, alors à ce moment-là on met des espèces de petites sandales avec des pneus et des trucs comme ça, à cause du rotin. Le rotin est une liane, plus grosse que le pouce, qui court sur le sol avec des épines horribles tous les dix centimètres. Et si on marche sur des épines 
de rotin, [ça fait mal]! Mais sur les pistes on va pieds nus. Elles ont été foulées par tellement de gens. Tout le monde est pieds nus, moi je suis encore pieds nus! Je me demande comment on peut se fourrer les pieds dans des chaussures qui font tellement mal. Le pied est comme la main, ça commence mince et ça se termine large. Et des chaussures, c'est juste l'inverse, ça commence large et ça termine mince. Alors votre pied dans une chaussure, c'est une absurdité totale!

\section{A. H. - Il y avait des voleurs?}

J.D. - Oui. Les Jörai en particulier étaient réputés pour être des voleurs merveilleux. Ils volaient des chevaux. Jamais de riz! C'est sacré, on n'a pas le droit d'y toucher. Les greniers à riz sont à l'extérieur du village, personne n'y touche jamais. Si jamais il y avait eu un vol de riz, ça aurait été un tollé, ça aurait été la mise à mort, ça aurait été effroyable. Je n'ai jamais connu de cas de vol de riz. Mais en dehors de ça, ça volait, oui, ça volait! Copieusement.

Tenez, j'avais une case qui n'avait pas de porte. Il m'arrivait de partir trois ou quatre jours en tournée. Ça restait grand ouvert, on ne m'a jamais rien volé. J'ai fait exprès même une ou deux fois de laisser quelques piastres vietnamiennes en vue comme ça sur mon bureau, qui était par terre. Quand je rentrais au bout de quatre ou cinq jours, on n'avait rien touché. On était rentré, seulement pour faire la poussière - des amis qui venaient nettoyer - oui! Ils ont vu, ils ont laissé.

A. H. - Et sur les pistes?

J. D. - Oh, on n'est pas en Chine. Il n'y a pas de bandits, il n'y a pas de pirates, il n'y a pas de brigands, rien sur les pistes. Il peut y avoir des tigres, au plus. Enfin le tigre est tellement froussard... Quand il sent l'homme, il fout le camp. Il faut vraiment qu'il soit affamé pour attaquer. En gros il n'y avait aucun danger, jamais d'attaque. C'est le pays le plus calme du monde.

A. H. - Les montagnards participaient à l'économie des Viêts?

J.D. - Oh, très localement, sur les petits marchés. Ils amenaient une hotte d'oranges pour avoir en échange un petit corsage, des trucs comme ça. Évidemment au sens large du terme, ça rentre dans l'économie, mais c'est tellement insignifiant dans le commerce vietnamien. Autrefois, c'était plus important, quand il n'y avait pas d'Européens, pas de commerce extérieur à la péninsule Indochinoise. Alors là, les Vietnamiens, qui n'étaient encore que sur la côte, avaient beaucoup d'échanges avec les populations de l'intérieur, qui descendaient avec des cochons, avec des défenses d'éléphant, avec des cornes de rhinocéros, avec du bois de cannelle pour avoir du poisson, du nước mắm, des trucs comme ça... Ça fonctionnait beaucoup.

Un peu plus tard, il y a eu quelques colporteurs annamites - c'est comme ça qu'on les appelait à l'époque - qui montaient sur les plateaux pour vendre des bricoles dans les villages. J'en ai vu.

À un moment, tout à fait de mèche avec les Viêt-minh, les colporteurs qui passaient partout ont vendu des bonbons empoisonnés, de l'alcool empoisonné. Pour 
les liquider! Parce que tous les Vietnamiens de tous bords, de Saïgon, de Hanoï, du maquis ou du gouvernement n'avaient qu'une idée: se débarrasser des montagnards. Ça, ça a duré assez longtemps. Il y a eu des centaines de morts chez les Jörai.

A. H. - C'était quand?

J. D. - Dans les années 1968, par là. Je suis parti en 1970.

\section{0}

J.D. - [Une chose qui serait intéressante à] étudier, c'est l'économie vietnamienne, culturellement vietnamienne en tant que telle, avant toute intrusion des étrangers.

Quelle était l'économie vietnamienne avant 1859? Dans les grandes lignes, il y avait la commune, les mandarins et l'empereur, un système confucéen. Il faut bien connaître l'aspect économique du confucianisme. Ce n'est pas si évident que ça, mais sans ça on ne peut rien savoir de l'économie vietnamienne avant l'intrusion des étrangers. Et encore, parce que si la prise de Saïgon remonte à 1859, il y a eu sous Louis XIV des Français qui se sont introduits et avant, il y avait des Portugais. [D'accord,] les Français étaient nuls pour le commerce et juste bons à faire des forts à la Vauban, mais les Portugais s'y entendaient davantage. Ils ont donc dû profondément - les Portugais surtout - modifier l'économie vietnamienne (ouverture de ports, etc.).

Il faut remonter plus loin encore, au XII ${ }^{\mathrm{e}}$ siècle à peu près, avec l'arrivée des Arabes. Une bonne partie du Champa est devenue musulmane ; la côte était commercialement colonisée par les Arabes. Ça remonte très loin, cette histoire-là. Donc, l'économie vietnamienne - si jamais il y en a eu une originale (parce qu'avant elle était chinoise) - a été modifiée par tous ces apports étrangers. Pour se débrouiller là-dedans, il faut être vraiment économiste, historien...

Je ne peux rien vous dire de plus, je vous ai dit tout ce que je savais, c'est-à-dire rien! Ce n'est pas mon problème, je n'ai jamais travaillé là-dessus.

A. H. - La seule économie vietnamienne, c'est l'économie villageoise?

J.D. - On peut l'étudier à ce niveau. Parce que, pas plus les Chinois que les Arabes et par la suite les Portugais et par la suite les Français n'ont pénétré les villages. Ils ont contacté les mandarins, les empereurs - les grands chefs - mais rien au niveau du village. S'il est vrai que le Viêt-nam est un pays de villages, l'économie du village est peut-être une clé pour comprendre... la victoire de 1975: [Vous pouvez détruire autant de villages que vous voulez], il y en a toujours un autre juste à côté. C'est comme dans les mythes. Vous coupez la tête du serpent et il en pousse sept nouvelles. C'est une des forces du Viêt-nam, parce que malgré toutes les apparences, mandarinales, impériales, confucianistes, c'est relativement anarchique: il n'y a pas de pouvoir 
supérieur, chacun se débrouille dans son coin. On ne parlait pas de l'empereur, on ne connaissait même pas le mandarin. Et l'empereur n'était au courant de rien, le mandarin non plus - et il s'en moquait. Pour une rébellion, pour le Viêt-minh, c'était la perle, la trouvaille. Ils ont joué là-dessus et c'est ça qui les a fait gagner. Quand un système comme le stalinisme nationaliste hanoïen peut s'infiltrer là-dedans, il y a des points partout sur la carte du ravitaillement des troupes. Il n'y a personne qui va le contrecarrer par pouvoir. Le petit village jouait sur l'anarchie.

Il fallait un Vietnamien comme Hô Chi Minh pour le sentir. C'est vraiment génial et ça a fonctionné. On doit aussi mettre cela en relation avec d'autres phénomènes d'impérialisme stalinisant, comme en Russie d'ailleurs. Chacun se débrouillait dans son petit coin, il n'y avait pas de pouvoir central: des petits sous-fifres secondaires [collectaient] des impôts qui restaient dans la poche des sous-fifres, mais le tsar n'était au courant de rien.

J. D. - Ce qui me met peu à l'aise, c'est que non seulement je ne suis pas économiste - je ne connais rien à l'économie - mais que ça ne m'intéresse absolument pas. Ce qui m'intéresse, c'est la culture, les techniques, la littérature orale, les mythes et les gens, les épopées, tout ce que ces gens racontent et ce qu'ils peuvent penser. Par-dessus le marché, les Vietnamiens, je n'y connais rien. J'ai vécu au milieu d'eux mais j'étais dans ma coquille: les Vietnamiens, la guerre, je m'en foutais. Que les Viêts, les Français, les Américains, les Chinois, les n'importe quoi se battent entre eux, j'étais complètement en dehors de ça. J'étais absolument le type des minorités.

Mon intérêt principal, c'était la culture. Je parle de valeurs intellectuelles, religieuses, mystiques, poétiques qu'on trouve dans la littérature orale, les mythes, les légendes, les poèmes. C'est là-dessus que je veux surtout travailler. Le reste je m'en fous. L'économie je m'en fous - ça, c'est mon économie. Tout ce que j'ai écrit sur les populations parmi lesquelles j'ai vécu et sur lesquelles j'ai travaillé va dans le sens que je viens de vous dire: des valeurs culturelles de qualité universelle.

A. H. - Mais les valeurs des gens ont quand même un regard sur l'économique. Quand on parle des valeurs de la réflexion, ils réfléchissent à gagner leur pain.

J. D. - Dans le secteur que je connais, la littérature, il n'y a pas cette réflexion. On ne peut rien en tirer, à moins de faire dire aux textes ce qu'ils ne disent pas. Rien. C'est « torpeureux », c'est poétique, c'est du rêve, c'est de l'ailleurs, c'est de la grande poésie! C'est comme si vous me demandiez s'il y a une dimension économique à la Chanson de Roland. Ou encore pire dans le roman du Graal: l'économie moderne dans le roman du Graal, c'est à se taper le derrière par terre!

A. H. - On ne se préoccupe pas du quotidien, en fait. 
J. D. - Non seulement on ne s'en préoccupe pas, mais on fait exprès d'aller ailleurs.

A.H. - Pour s'échapper...

J.D. - Ce n'est pas pour s'échapper. C'est pour rigoler. Une échappatoire, ça voudrait dire qu'on voudrait fuir quelque chose. On n'a rien à fuir. Le quotidien, on le vit - entre rêves - mais ce n'est pas une valeur. Le riz, ce n'est pas une valeur. Et cuire du riz, ce n'est pas une valeur. C'est une nécessité, comme on va faire caca. Tous les jours, je bouffe mon riz, tous les jours je fais caca, tous les jours je bouffe mon piment. C'est des trucs mécaniques, biologiques. On s'en fout. C'est naturel, c'est simple, on n'en parle pas, ça n'a aucun intérêt.

Quand on est libre le soir, la nuit, ou quand il pleut trop, on ne peut pas aller se balader dehors: on boit! On invente un tas de belles choses, le Graal, la Chanson de Roland, Tristan et Iseult. Les côtés de l'économique là-dedans? C'est de l'antiéconomique justement. C'est du gratuit. C'est de la création pure et la création est gratuite par définition.

Alors les économistes !... Quand je vois Le Monde par exemple, je lis la première page, quelquefois la seconde, rarement la dernière. Mais quand je vois trois ou quatre pages «Économie » à l'intérieur, ça va immédiatement dans le poêle pour allumer mon feu. Je suis complètement allergique à l'économie. Parce que c'est exactement l'inverse de la gratuité - et la création littéraire c'est la gratuité. Ça ne sert à rien, ça ne rapporte pas un sou à tous ces gens-là d'avoir inventé des mythes extraordinaires. Au Moyen Âge, il n'y avait pas de droits d'auteur. Les auteurs de Tristan et Iseult, de la quête du Graal, du roi Arthur n'en ont jamais touché.

Quand je traduis leurs mythes et que je peux les publier, je leur envoie du fric. Mais ils n'y pensaient même pas. Ils ne comprennent même pas que d'avoir conté un truc merveilleux, ça peut rapporter du fric. Moi, c'est pour les remercier, parce que je n'ai jamais payé un informateur. Je n'avais pas d'informateurs, j'avais des amis. Je n'ai jamais donné un sou à qui que ce soit. D'ailleurs là-bas je n'avais pas un rond - et maintenant ça recommence, je n'ai pas un rond. Quand j'étais au CNRS, je pouvais un peu me débrouiller, j'ai publié. Alors de temps en temps, j'essayais de me dédommager, d'envoyer. Ils ne comprennent pas! C'est gratuit, on ne paie pas quelque chose qui est gratuit! C'est une injure presque: je vous fais un cadeau et vous me donnez de l'argent en échange de mon cadeau. Un conteur qui me raconte une épopée magnifique qui dure quatre ou cinq heures, ce serait une injure que de lui donner quoi que ce soit. C'est la giclée de la gratuité.

Donc je suis antiéconomique comme je suis anarchiste! Je suis comédien, vous savez. Je sais très bien que j'ai joué la comédie. Je fais exprès, je sais ce que je fais, ça m'amuse et en même temps, il y a quelque chose à en tirer.

L'économie et l'argent, il ne faut pas m'en parler. Je suis de l'autre côté, carrément de l'autre côté. Et ça ne vient pas de mes racines culturelles : je suis fils de grand 
bourgeois et j'avais beaucoup d'argent. C'est venu au contact des sauvages. C'est eux qui m'ont cultivé. Oui! Ils m'ont appris des valeurs que j'ignorais... On peut être dans la boue de la rizière, on peut faire des travaux idiots qu'on n'aime pas, il faut bien bouffer. Et il y a un moment où ça sort et on est libre et on est soi et puis on invente, on crée, on fait des poèmes. Ils m'ont révélé ça: qu'on pouvait créer, en sortant de sa merde, tout en y étant toujours. Et ça, c'est fort.

Pour moi, ces populations ont plus d'attrait. On les tue bien sûr. On fait tout pour les tuer. On a tellement peur de la création, parce que la création est anarchique, par définition. On crée, donc on sort des normes, donc c'est anarchique, alors ils suppriment. Le Viêt-nam le fait avec ses minorités et la Russie l'a fait avec des milliers de peuples jusqu'au fin fond de la Sibérie, pour les liquider et les remplacer par les Slaves. Ils ne sont pas comme les autres, ils inventent, ils sont artistes. En [les] supprimant, on supprime tous ceux qui ont le culot, le toupet, l'audace de vouloir créer, sortir - pas s'évader - sortir, monter, aller voir autre chose.

Vous voyez, dans un village jörai, une quinzaine de personnes dans la case. C'est spontané. Un conteur se met à commencer une épopée. Il exprime un «non encore pensé » qui était un peu chez tout le monde et qu'il fait surgir comme ça, par son organe : la voix! C'est un comédien, un conteur. Et l'organe, il faut l'entendre. J'ai des enregistrements d'une beauté, par la langue, par son organe, qui charme. Et il dit ce qu'il y a en dessous, ce qu'il y a dedans, ce qu'il y a dans tous les cœurs qui sont là, les gosses comme les vieux. Au bout d'une heure de conte, les gosses s'endorment, parce qu'il n'y a pas de conte pour enfants, il n'y a pas de conte pour vieillards. Tout le monde est là, tout le monde prend ce qu'il peut. Les gosses sont endormis, les vieux un peu plus tard, les femmes les dernières : elles sont coriaces, elles ne dorment pas beaucoup. Il y a des conteuses aussi. Ça, c'est extraordinaire.

C'est ce que j'appelle l'économie de ces populations-là.Je renverse complètement le problème, exprès! C'est leur économie. Ça marche encore. Il y a vingt-deux ans que je les ai quittés, ils m'écrivent encore. Dans la dernière lettre, ils disaient: « Vous vous intéressiez vachement à nos contes. Nous aussi on s'est mis à s'y intéresser. Si vous en voulez, on va vous envoyer encore des textes ». Ce n'est pas génial, ça?

L'économie, ce n'est pas les sous, ce n'est pas le grain de riz, ce n'est pas l'huile d'arachide. Oikos, c'est la maison. Nomia, c'est la règle. C'est du grec. Oikosnomia, l'économie, c'est la « règle de la maison ». Chacun a ses règles. Les Romains, c'est des épiciers. Les Grecs étaient des tragédiens. Les Jörai et d'autres sont des conteurs de légendes, des artistes et des musiciens. C'est leur économie. C'est comme ça que chez eux se règlent les affaires, les différends de justice et tout ça, c'est génial. On chante, on récite en chantant des versets : " s'il y a ceci, il y a cela - s'il y a cela, il y a ceci, etc., etc. ». Ça dure des heures, c'est tellement beau qu'à la fin tout le monde dit d'accord et le différend est réglé. C'est fini, on n'en parle plus, pas de prison, pas de juge, pas d'amende, pas de dette, rien. On boit un bon coup et puis c'est fini. Parce qu'on a fait ça avec leur oikosnomia, avec leur règlement de la maison, par la 
voix, par la parole, par invention en raboutant des textes, à qui parlera le mieux. Et c'est ça mon économie.

Ça va loin ce que je dis: essayez de vous pencher un peu sur l'économie des gens qui n'ont pas d'économie au sens moderne, occidental, du mot. Il y a un bouquin à faire là-dessus.

Économie de mots aussi. On va jouer sur les mots, en comédien. Avec trois mots on vous exprime une foule de choses, parce qu'il n'y a rien de plus condensé que la littérature orale. Il ne faut pas croire que ce sont des trucs qui se répètent et se répètent, qui se rallongent, qui se raboutent. En trois mots, on vous dit plein de choses. Un exemple: une devinette en quatre mots, quatre monosyllabes. «Âko' hơoñ tung čum ». Âko', la tête - hơoñ, caresse - tung, le cul - čum, baiser. C'est splendide! C'est la pipe. Le dernier gosse jörai sait ça. Comme économie de moyens, [c'est fort] hein? Économie de paroles, économie de mots. Vous n'avez pas pensé à cette dimension de l'économie... Ce n'est pas génial?

J'ai tout appris là-bas. Qu'est-ce que vous voulez, j'avais de bons profs quand j'étais au lycée mais jamais à ce degré-là. Ça fait revoir toutes les notions qu'on avait avant. Quatre monosyllabes qui jouent sur les mots bien sûr, parce qu'on veut que ce soit choquant. Quel humour, quelle économie, quatre monosyllabes! Il y a toute une philosophie là-dedans. Ça veut dire, « baiser, caresser, culotter, c'est la rigolade - une bonne bouffée, ça vaut mieux ». Il y a ça aussi dedans. Non, je n'avais jamais appris de trucs pareils.

0000000000

J. D. - Je travaille sur l'architecture... Regardez encore la construction : comme économie de moyens, ce n'est pas cochon. Quelques bouts de bambou, quelques bouts de bois et on fait une maison qui résiste à toutes les tempêtes. Et ça, je l'ai vu dans un village. Les maisons sur pilotis. À côté de ça, les Vietnamiens avaient des cases en pisé, au ras du sol. Un typhon : les Vietnamiens... plus rien!

A. H. - Et c'est ça l'économie aussi, la forêt?

J.D. - Bon, là encore, j'en aurais long à dire, très long à dire, parce que je me suis battu avec des botanistes, pédologues, géologues, tous les «-logues » que vous voulez.

La technique de l'essartage, ça suppose une faible densité de population au kilomètre carré. Donc c'est un grand luxe, qu'on peut se permettre dans des régions où il n'y a personne. En quoi ça consiste? À délimiter un périmètre de forêt, à abattre d'abord la broussaille, ensuite à abattre les gros arbres qui en tombant écrasent tout ce qu'on avait à moitié abattu avant et à bien nettoyer. On y met le feu, avec des pare-feu tout autour (j'y ai assisté, j'y ai participé!). Le feu ne passe pas à un mètre 
des pare-feu. Des pare-feu parfaits. On met le feu là-dedans. C'est au mois d'avril, à la fin de la saison sèche. Parce qu'en mai, il commence à pleuvoir un petit peu. Sur les cendres, avec le bâton à fouir - fouir c'est faire un trou - on met des grains. Du riz, du maïs, des courges, tout mélangé. Là-dessus - fin mai, juin, juillet, août, septembre même - il pleut. Il faut juste désherber un petit peu. Octobre, c'est à peu près la fin des pluies. Novembre, on récolte, on récolte, on récolte, de quoi bouffer pendant presque une année. Du vachement bon riz, pas la cochonnerie que vous avez bouffée à Hanoï. Le riz d'essart, c'est le meilleur possible. Vous savez que le riz, c'est une plante normalement aquatique, mais depuis je ne sais pas combien de siècles, sinon des millénaires, il s'est acclimaté à des terrains secs qui reçoivent beaucoup de pluie. Cet essart, on le cultive pendant deux ans, trois ans maximum, parce qu'après la terre va s'épuiser.

Je vous donne un exemple. Dans le village de Choreo en pays jörai, où j'étais, j'ai construit ma maison sur un ancien essart, abandonné depuis dix ans. Il y avait des arbres de vingt centimètres de diamètre. Il ne faut pas dire que la pratique du brûlis détruit la forêt, ça pousse comme du chiendent. J'ai abattu un certain nombre de ces arbres pour faire ma maison et pour les remplacer par des arbres fruitiers. J'ai enlevé des espèces qui n'étaient pas utiles et j'ai mis des arbres fruitiers à la placedes mangues et des trucs comme ça. Le brûlis ne détruit pas du tout.

Donc voilà l'économie de forêt de ces populations. On coupe rigoureusement ce qu'il faut. C'est la même chose pour couper un arbre pour en faire une maison. On ne va pas en couper dix si on en a besoin d'un. On en coupe un. Quand on va à la chasse, on ne va pas tuer dix cerfs comme font les Français. Ils ne peuvent même pas les bouffer, ça pourrit. On en tue un à la rigueur, pour nourrir tout un village pendant un mois. C'est économique - et au sens ancien du mot. L'économie, c'est tout, parce qu'on n'a rien, on est pauvre. Les grands économes sont des pauvres. Des vrais pauvres, pas les pauvres qui vont au supermarché et qui achètent à crédit, même les bagnoles. Même les gosses ont chacun leur bagnole, trois ou quatre bagnoles par maison. Ce sont de faux pauvres.

Alors à côté de ça, l'économie américaine au Viêt-nam! Pour eux, la forêt, c'est là que sont tous les Viêt-minh. D'abord du défoliant, très dangereux pour les hommes. J'ai eu un copain missionnaire qui a été grièvement brûlé, immobilisé pour la vie, simplement parce qu'il en a reçu une giclée. C'était un avion à haute altitude. Dans mon jardin, à plus de vingt kilomètres du passage de l'avion, j'ai eu du maïs qui était crochu comme ça. À vingt kilomètres. C'est ça, l'économie américaine : un bon passage de défoliant, puis il ne sortira plus rien. Là-dessus : napalm. Je l'ai vu, j'ai des témoignages, j'ai même des rapports officiels de l'ONU. Il faudra plusieurs siècles avant que la forêt indochinoise puisse repartir - si elle repart jamais.

Les Américains ont commencé à désertifier le Viêt-nam et les Vietnamiens ont continué! Quand les Américains sont partis comme des lapins, ils ont tout laissé, les bulldozers comme les avions. Les Vietnamiens ont tout ramassé et ils ont continué. 
Sur ce qu'il reste de cette forêt, ils ont passé le bulldozer. Je l'ai vu ça aussi. Des jardins moï - pas des jardins vietnamiens - passés au bulldozer. Vroum, on arrache tout! Quand ces braves montagnards faisaient des essarts ou des jardins, ils laissaient toujours par-ci par-là un arbre [contre] l'érosion, pour retenir le sol (dans ces zones tropicales, il y a peut-être vingt centimètres de terre cultivable). Mais maintenant, on démolit tout! Dans dix ans, le Viêt-nam sera le Sahara et les montagnards n'auront plus qu'à crever.

A.H. - Qu'est-ce qu'ils font, les montagnards?

J.D. - Ah, ils se plaignent. Ils ne peuvent rien faire: ils ne sont reconnus par personne.

A. H. - Je veux dire, économiquement, pour manger.

J. D. - Ils font des petits jardins.

A.H. - Ils ne peuvent plus faire des essarts?

J. D. - Il n'y a plus rien à brûler : ce qui n'a pas brûlé a été rasé au bulldozer. Parce que pour le Vietnamien (comme les Russes, ils ont piqué ça aux Russes), l'économie c'est le rendement immédiat.

C'est comme ça qu'on a tué la mer d'Aral et j'ai vu le même coup au Viêt-nam, à vingt kilomètres en aval du village où j'étais, sur le grand fleuve Apa. Il y avait des géologues, des Hollandais, un tas de gens - c'était entre 1954 et 1975 - venus pour étudier des problèmes, pour faire des champs de coton. Ils ont repéré un coin au bord du fleuve pour faire le coton. Chose curieuse, il n'y avait aucun établissement de Jörai là-dessus.

Contre tous mes avis, contre l'avis des experts amis jörai, le gouvernement a décidé de faire un immense champ de coton. On installe des Vietnamiens, on installe plein de maisons, des champs. Quelques mois après, crue du fleuve! Les Jörai ils le savaient, c'est pour ça ils n'ont jamais même fait un jardin là-dessus. Le coton, les maisons, les Vietnamiens, tout est parti vers la mer. Et voilà, liquidé. Ça, c'est un procédé russe, c'est un procédé vietnamien, c'est un procédé mondial. C'est ce qu'on appelle l'économie!

Alors que moi,j'ai le culot de soutenir l'économie des sauvages... qui protègent la nature. Ils ne détruisent pas pour détruire, ils détruisent juste pour vivre. Exactement comme le tigre (il n'y a pas plus malin que le tigre): s'il a besoin d'une proie, il en bouffe une, pas dix. Il détruit juste ce qu'il lui faut, pas plus. Et par-dessus le marché - alors ça, c'est le comble du comble! - quand un Jörai abat un arbre, il lui demande pardon avant! Voilà, j'en suis là. On taille un rosier, on demande pardon au rosier de couper une branche. J'ai peur de faire mal. Ça, c'est sauvage. Ça, c'est économique.

A.H. - Reste-t-il des montagnards, des peuples au Laos, en Chine, en Birmanie qui pratiquent toujours l'essart?

J.D. - Ça va être difficile de répondre. Depuis trop d'années je suis coupé de tout renseignement. 
Le Laos est maintenant devenu une colonie vietnamienne parce que les Vietnamiens s'estiment les héritiers de l'Indochine française. Vous savez, j'ai vu dans les années 1960 des cahiers d'écolier des écoles vietnamiennes (dans la région gouvernée par Saïgon, pas par Hanoï). Dans ces cahiers d'écolier, il y avait une carte du Viêt-nam [qui regroupait] Viêt-nam, Laos et Cambodge [sous] la même couleur !

Quand les Français sont allés s'installer au Laos et au Cambodge, ils ont pris des Vietnamiens avec eux. Ce sont les Français qui ont introduit les premiers Vietnamiens au Laos, les premiers Vietnamiens sur les hauts plateaux. Parce qu'il était impensable pour un Français d'avoir comme chauffeur de son automobile ou comme cuisinier un sauvage aux pieds nus. Il lui fallait un Vietnamien bien civilisé, bien policé.

Maintenant les Vietnamiens ont bouffé le Laos, complètement. Ils étaient sur le point de bouffer le Cambodge, quand il y a eu l'affaire Pol Pot. [Puis il y a eu la] conquête militaire par le Viêt-nam et le régime de collaboration de Heng Samrin. Maintenant il y a Norodom Sihanouk, le grand comédien, qui essaie de revenir sur la scène. Je ne peux pas vous dire ce qui se passe au Laos ou au Viêt-nam. Sinon que bon, l'essartage est interdit et le bulldozer est toléré. Voilà l'économie!

Alors les populations véritablement économes, les autochtones... Là encore il y a un truc: " autochtone », ça veut dire les gens qui sont de la terre. La terre c'est la leur, ils la respectent. Il n'y a pas de document écrit disant que c'est à eux, mais depuis des millénaires c'est à eux. Ils le savent et ils lui rendent grâce, à la terre. Elle les nourrit, ils la respectent. La politique des uns, c'est de respecter leur terre. Et la politique des autres - les économistes mondiaux - c'est de la leur arracher. Il n'y a pas d'écrits, pas de textes : cette terre est à personne. Et donc le gouvernement, le pouvoir en place veut s'en occuper. En particulier les Vietnamiens qui arrivent sur les hauts plateaux. Ce n'est pas leur terre, ils s'en foutent et ils détruisent tout, ils cassent tout. Ils coupent tout, bousillent tout, pendant un an ou deux, pour faire des légumes, des choux, etc. - rendement oblige - et abandonnent tout après quelques années. C'est authentique, je l'ai vu.

Pour [les colons] français, c'était l'opposé: le Viêt-nam, l'Indochine, c'était leur terre. Par droit de conquête ou tout ce qu'on veut, mais c'était à eux. Ils la respectaient, la terre. Ils ne détruisaient rien. Il y a opposition complète entre les Français et les Américains. Le Français en faisait sa terre: beaucoup de colons et de planteurs étaient des paysans! Ils savaient ce que c'est que la terre et que la terre, on la respecte la terre, on ne la détruit pas. Pour les Américains, il n'y a pas de terre, il n'y a pas de peuple, il n'y a rien. Il fallait faire table rase, pour faire un no man's land entre les Philippines et la Chine, pour qu'ils aient des points d'appui solides dans le Sud-Est asiatique. Pour le Français, c'était sacré. J'en ai rencontré beaucoup de ces colons. J'en ai croisé des impossibles, des petits colons [qui abusaient sans vergogne] des montagnards, coolies et tout! Mais c'était leur terre...

A. H. - Ils s'y étaient investis. 
J.D. - Enracinés! Enracinés! Un fonctionnaire restait deux ans, trois ans maximum. Le colon restait toute sa vie, tous les dix ans un retour en France à tout casser. Ça montre le respect de la terre.

L'autochtone, les indigènes, les aborigènes, c'est leur terre, ils la respectent. Mais les colons vietnamiens d'aujourd'hui, ceux qui envahissent toutes ces zones, [c'est une autre histoire]... Dans la vallée de Dran, où il y avait trois ou quatre mille montagnards, il y a eu du jour au lendemain 300000 Vietnamiens. Oui ! J'ai des chiffres, j'ai tout, j'ai des documents. Ce n'était pas leur terre, ils ont tout détruit. Rendement, rendement, rendement! Et maintenant la terre est usée, il n'y a plus rien, ils crèvent de faim. Trop pressés. Le vrai paysan n'est jamais pressé. On ne tire pas sur une plante pour qu'elle pousse, on attend. C'est simple comme bonjour. En plus, le Vietnamien a la haine des arbres. Le Vietnamien, c'est un maraîcher, un riziculteur et un maraîcher: table rase, tout est tout propre, tout net.

A. H. - Dans les deltas, il n'y a pas d'arbres?

J.D. - Nulle part! Nulle part, en pays vietnamien. Ils ne peuvent pas supporter un arbre. S'il y en a un, ils l'abattent. Comme il faut que ça rende le plus possible, on s'arrange pour que tout soit comme une rizière. Ils ont tellement bien fait que quatre-vingt-dix millions de Vietnamiens crèvent de faim. Et ça explique les boat people: ils essayaient de foutre le camp, parce qu'ils n'en pouvaient plus de crever. Il n'y a aucune raison politique, aucune. Parce qu'ils se foutaient éperdument du marxisme - je parle des gens de Hanoï. Ils s'en sont servis pour avoir l'aide russe, le matériel, pour avoir des slogans, la langue de bois, etc. mais c'était le nationalisme, le patriotisme et tout ce qu'on veut, récupéré.

Je sais que tout le monde ne peut pas vivre du régime de l'essartage. C'est un régime de luxe, pour une densité de huit à neuf au kilomètre carré. Aux grandes densités, ce n'est pas possible. [Mais] entre l'essartage de luxe et la désertification, il y a peut-être une solution.

\section{$\infty 00000000$}

J.D. - Vous avez rêvé cette nuit?

A. H. - Je n'en suis pas conscient.

J.D. - Tout le monde rêve, même les chiens. Moi, depuis longtemps, j'ai pris l'habitude de la technique de mémorisation des rêves au fur et à mesure. Et les plus intéressants, je les note. J'en ai fait une dizaine de cahiers, parce qu'il y en a eu de très intéressants. C'est très important pour se connaître.

Les rêves, je les ai étudiés là-bas, chez les montagnards. Il y a des rêves qui quelquefois les inquiètent et pour s'en débarrasser, il faut qu'ils les racontent à quelqu'un. Surtout chez les Jörai, j'avais des femmes qui venaient me raconter leurs 
rêves le matin. Pas un ethnologue qui a eu une chance pareille! Elles me les racontaient; comme ça, ça les débarrassait. Il n'y avait pas d'effets mauvais et c'est moi qui ramassais tout. Théoriquement pour elles, j'étais plus fort, je pouvais ramasser tout ça et puis elles étaient libres. Il y a un tas de choses là-dedans.

A. H. - Comment ils sont, ces rêves?

J. D. - Il ne faut jamais interpréter, il faut les prendre comme ça. C'est comme un morceau de musique: faut pas dire que ça signifie telle chose, on l'écoute, ça produit un certain effet et c'est tout. Il ne faut pas interpréter comme un tableau. On reçoit, voilà.

Le rêve, c'est toujours extrêmement court. J'ai des bouquins de récits de rêves, des gens qui font une page pour une petite histoire qui a duré un quart de seconde. Pour mémoriser ça, il faut prendre un mot-clé ou une image clé, ou une couleur ou quelque chose que vous attrapez. Au réveil vous vous fixez sur le mot-clé et tout le rêve revient. Ça tient en trois lignes... Plus de la moitié des possibilités de notre cerveau fonctionnent à l'état de rêve. À l'état de veille, nous n'utilisons que très peu de nos possibilités. Les gens qui s'imaginent peu doués, sans imagination, banals, quand ils se mettent à faire attention à leurs rêves, ils s'aperçoivent qu'ils ont des richesses fantastiques, de l'imagination débordante. Souvent c'est la culture du milieu familial ou autre chose qui bloque.

C'est comme pour la langue. Vous savez que les petits enfants du monde entier, les bébés, arrivent à prononcer toutes les choses qui sont prononçables dans le monde. Il y a un cas particulier que j'ai étudié : c'est la glottalisée. Les consonnes glottalisées /'bo/, /'be/, /'de/. Très peu d'Européens peuvent les prononcer, très peu. Les Vietnamiens n'en ont que deux, /'be/ et /'de/.C'est pour ça ils vous disent « 'bonjour » et non pas « bonjour ». Le « $b$ » implosif: «'bon, 'bonjour ». Et j'ai entendu des petits enfants français, des bébés de deux ans environ, faire des glottalisées magnifiques. L'éducation, le fait d'entendre la langue des parents, ça limite, ça limite, ça limite de plus en plus les moyens d'expression pour se faire comprendre. Il y a eu des possibilités énormes. L'éducation les met sur des rails, il ne reste plus grand-chose.

Ce que je vous dis sur la langue, c'est comme ce que je vous disais sur le rêve. Il y a un point commun: c'est le cerveau. Le cerveau, qui commande le langage. À la naissance, les cerveaux sont capables d'un tas de choses. Même avant. C'est pour ça que c'est très important qu'une femme enceinte entende de belles choses, même de la musique. Oui, très important pour l'enfant dans le ventre de la mère. Il y a des mères qui font ça très bien, elles parlent et ça reste. Il y a de la mémoire de ce qui s'était passé avant la sortie du ventre. Ça existe, la mémoire de l'état fotal.

A. H. - Est-ce que les montagnards se racontent les rêves?

J.D. - Entre eux? Dans la vie courante, non. Habituellement pas. [Ou quand ils le font,] c'est à une personne seulement et pour s'en débarrasser, comme je vous l'ai dit. Mais dans la mythologie, dans la littérature orale, oui. Il y a le héros, qui est 
jeune homme et qui a un copain qui raconte qu'il a vu en rêve une belle jeune fille, qu'il lui prenait les seins, etc., etc. Alors les autres lui disent: « Fais nous rencontrer ta belle! », etc. Voilà le genre de ce qui peut se produire en littérature. On raconte pour que ça entraîne l'histoire.

A.H. - Technique littéraire plutôt qu'autre chose?

J.D. - Oui, mais on ne peut pas distinguer le littéraire du courant pas plus qu'on ne peut distinguer le sacré du profane. Chez eux, tout a un caractère sacré et chez eux tout a un caractère littéraire. Ce sont des littéraires, ces paysans, ces sauvages... ce sont des littéraires! Dans la conversation courante, ils s'arrangent pour faire des assonances, des allitérations, des rimes. Ils ne peuvent pas s'en empêcher. Ce sont des maîtres de la parole, c'est extraordinaire. J'ai des milliers de pages de textes enregistrés puis transcrits, pas tous traduits encore, c'est délirant. Et tout le monde aime ça, les jeunes comme les vieux. Alors c'est bien simple, à l'école, ils sont nuls en maths, ils sont très forts en lettres. L'inverse des Vietnamiens, qui sont des matheux, des ingénieurs, des industriels et qui ne sont pas très forts en lettres et en philo. Ils n'ont pas de littérature, les Vietnamiens, presque pas, rien d'intéressant. C'est juste l'inverse.

A.H. - Et les contes, ça se raconte comment chez les Jörai?

J.D. - Ça dépend de l'âge. Les jeunes qui commencent à s'exercer racontent n'importe quoi, n'importe quand, n'importe comment, à n'importe qui. C'est facile pour l'étranger qui débarque, qui sait un petit peu la langue et qui commence avec les gosses. On apprend beaucoup de choses déjà. On sait qu'il y a une histoire, qu'il y a un type qui a un nom de héros, etc. Et puis au bout de quelques années quand on se débrouille mieux dans la langue, on arrive à trouver quelqu'un qui vous raconte ça pendant quatre ou cinq heures de suite. Parce que le gosse, ça dure dix minutes. C'est comme Cendrillon ou Le Petit Chaperon rouge. Vous demandez à un gosse de vous le raconter, ça durera trois minutes. S'il y a encore quelques vieux conteurs, vieilles conteuses en France, ils vous racontent ça pendant des heures.

Ce n'est pas du tout la même circonstance. Pour l'enfant il n'y a pas de circonstance. Pour le conteur, il doit y avoir des époques de l'année: des époques où il n'y a pas de travail à la rizière et quand il pleut. Puis si ça leur prend envie - il n'est pas question de leur demander -, le soir, la nuit, dans leur maison, la maison du voisin ou celle des copains chez qui on va passer la soirée, ils font un conte. Ça peut durer une heure, ça peut durer cinq heures, jusqu'à l'aube... Et vous avez ça dans tout le Sud-Est asiatique. Le reste du monde je n'ai pas étudié.

Il n'y a pas de rite particulier. Il y a simplement une époque de l'année, une heure de la journée, [quand il y a moins de travail et qu'on peut veiller un peu tard]... Parce que vous savez, quand vous travaillez dans la rizière, c'est à sept heures et demie qu'on va se coucher. Quand on ne fiche rien de toute sa journée, parce qu'il pleut trop, ou parce que le riz vient d'être moissonné tout simplement, il n'y a plus rien à faire. C'est la meilleure époque, de mi-décembre à fin janvier. Alors ils n'ont rien à ficher. Les travaux de réfection de la maison, ça se fait plutôt à partir de février. 
La préparation des champs se fait en mars-avril. Alors là, à la fin de l'année, décembre-janvier, c'est plus facile d'entendre des contes. C'est spontané. Ils sont vraiment bordéliques, s'ils ont envie, ils racontent, c'est tout.

A. H. - Il n'y a pas de conteurs professionnels?

J. D. - Non, pas de professionnels. Ce n'est pas du tout comme en Afrique avec le griot. Ils ne se font pas payer. Ils le font pour le plaisir. Ils ne boivent pas, jamais avant de conter - ça abîme la voix - ils boivent après. Et comme, vous savez, ils ne sont pas très sobres: quand ils boivent, ils boivent trop et ils ne peuvent plus raconter. Ils le savent très bien.

A. H. - Et ils content uniquement avec la voix...?

J. D. - Il n'y a pas de musique. Beaucoup sont aussi musiciens, tout le monde est musicien. Presque tout le monde a un instrument de musique qu'il fabrique lui-même. Ceux qu'ils fabriquent eux-mêmes sont des vents et des cordes - flûte, pipeau et genre cithare. Presque tous jouent aussi d'un instrument mais il n'y a jamais d'accompagnement avec le conte. Il n'y a pas de gestes, ils sont assis.

A. H. - Beaucoup de gens?

J. D. - Oh non, une dizaine de personnes dans la case. On ne fait pas de rassemblement pour ça. Compte tenu que c'est imprévisible, on ne sait pas quand il va conter. J'avais des amis là, dans le village, qui disaient: « Il y a un tel qui est conteur, qui va passer la soirée chez nous. Il va peut-être conter, je n'en sais rien. » Il y en a quelques-uns qui sont venus conter chez moi, pour me faire plaisir, tout seul.

A.H. - Pouvez-vous me donner un exemple d'un conte?

J.D. - Beaucoup... J'ai appris à être conteur...

À Cerisy... Je ne sais pas si vous connaissez les colloques de Cerisy: c'est un château en Normandie qui a un centre culturel et où chaque été il y a une dizaine de colloques sur des sujets très précis. J'avais été invité - il y a sept ou huit ans au moins - pour un colloque sur le mythe et la mythologie. J'ai fait une conférence et j'avais demandé, dans ce très grand hall du château, d'avoir la possibilité de conter le soir. Alors on avait préparé le grenier du château, une vraie cathédrale, une splendeur! Il y avait une cinquantaine de personnes. J'avais demandé qu'on nous serve des boissons: on nous servait des punchs, des trucs comme ça. Je me suis mis à conter. J'ai conté un tas d'histoires, ça a commencé vers dix heures du soir à peu près, juste après le dîner. À minuit ils n'en pouvaient plus mais ils m'en demandaient encore. Et de mémoire! Je n'avais aucun texte, je n'avais rien apporté: je ne pensais pas du tout conter, je pensais faire ma conférence - je l'avais dans la tête, la conférence - et puis c'est tout. Et quand je me suis dit «mais ils aimeraient peut-être que je conte? », je me suis promené dans le parc du château toute la journée avant cette soirée-là, pour me remémorer - sans aucune note, sans rien - pour me remémorer de jolies histoires. Puis hop, c'est parti ! 